\title{
Impact of gender and healthy aging on pulmonary capillary wedge pressure estimated by the kinetics- tracking index using two-dimensional speckle tracking echocardiography
}

\author{
Masanori Kawasaki ${ }^{1}$, Ryuhei Tanaka ${ }^{2}$, Koji Ono ${ }^{2}$, Shingo Minatoguchi ${ }^{1}$, Takatomo Watanabe ${ }^{1}$, \\ Masazumi Arai ${ }^{2}$, Kazuhiko Nishigaki ${ }^{1}$, Toshiyuki Noda ${ }^{2}$, Sachiro Watanabe ${ }^{2}$ and Shinya Minatoguchi ${ }^{1}$
}

Risk stratification in heart failure (HF) among patients and healthy subjects using pulmonary capillary wedge pressure (PCWP) is important for understanding when and why HF develops. The aim of the present study was to evaluate the impact of gender and healthy aging on estimated PCWP using a kinetics-tracking index in patients and in healthy subjects without hypertension. The study population consisted of 198 healthy subjects without cardiovascular or other systemic diseases and who were not taking any medications. Echocardiographic studies were performed using an ACUSON Sequoia 512 ultrasound system. Active left atrial (LA) emptying function (EF) was defined as (pre-atrial contraction LA volume - minimum LA volume)/pre-atrial contraction LA volume $\times 100 \%$. With an increase in age, the $\mathrm{E} / \mathrm{A}$ and $\mathrm{E} / \mathrm{e}^{\prime}$ ratios (markers of left ventricular (LV) diastolic dysfunction (DD)) showed a similar decrease in males and females. PCWP was maintained at $8.3 \pm 1.8 \mathrm{~mm} \mathrm{Hg}$ in males and $8.2 \pm 2.3 \mathrm{~mm} \mathrm{Hg}$ in females because of compensation by an increase in active LA EF. In contrast, the compensation for LV DD with an increase in active LA EF in females tended to be more gradual (slope $=0.11$ ) than in males (slope $=0.18, P=0.060$ vs. female). The parameters that indicated LV DD deteriorated with advancing age. PCWP might be maintained because of compensation, namely an increase in active LA EF in both males and females. The compensation in female septuagenarians and octogenarians was weaker than in male septuagenarians and octogenarians. This difference in compensation may explain why HF with preserved LV ejection fraction occurs more frequently in females than in males.

Hypertension Research (2016) 39, 327-333; doi:10.1038/hr.2015.149; published online 21 January 2016

Keywords: diastolic function; gender; healthy aging; left atrial function; pulmonary capillary wedge pressure

\section{INTRODUCTION}

Measurement of intracardiac pressure, such as pulmonary capillary wedge pressure (PCWP) or left ventricular (LV) filling pressure, is useful for the stratification of LV diastolic dysfunction (DD) that can result in heart failure (HF) because of hypertension and aging. ${ }^{1,2}$ However, measurement of PCWP or LV filling pressure is an invasive method, and there have been few noninvasive indices that can precisely estimate PCWP or LV filling pressure. Recently, we developed a novel index, the kinetics-tracking index (KT index) to predict PCWP based on the combination of active left atrial (LA) emptying function (EF) and minimum LA volume (LAV) index obtained from the time-LAV curve using speckle tracking echocardiography (STE). The PCWP estimated by the KT index (ePCWP) had a strong correlation with the PCWP obtained by right-heart catheterization $(r=0.92)$ in patients with normal sinus rhythm. ${ }^{3}$ Furthermore, the KT index was a more accurate and useful predictor of PCWP than E/e. ${ }^{3}$
There have been no previous reports evaluating PCWP in healthy subjects in a relatively large population because of the absence of a noninvasive method to measure PCWP in healthy subjects. Therefore, the aim of the present study was to evaluate the impact of gender and healthy aging on ePCWP and other echocardiographic parameters in patients and in healthy subjects without hypertension.

\section{METHODS}

Subjects and study protocol

The study population consisted of healthy subjects without cardiovascular or other systemic diseases and who were not taking any medications. Subjects who had a normal chest X-ray and echocardiographic findings according to the recommendations of the American Society of Echocardiography ${ }^{4}$ were included in the present study. We also included patients who had trivial valvular regurgitation and those who had abnormal values of diastolic function parameters, such as E/A (ratio of the peak early diastolic LV filling velocity to peak atrial filling velocity) and E/e' (ratio of peak early diastolic transmitral

${ }^{1}$ Department of Cardiology, Gifu University Graduate School of Medicine, Gifu, Japan and 2Department of Cardiology, Gifu Prefectural General Medical Center, Gifu, Japan Correspondence: Dr M Kawasaki, Department of Cardiology, Gifu University Graduate School of Medicine, 1-1 Yanagido, Gifu 501-1194, Japan.

E-mail: masanori@ya2.so-net.ne.jp

Received 24 June 2015; revised 19 October 2015; accepted 23 October 2015; published online 21 January 2016 
inflow velocity to annular tissue velocity). All subjects had normal findings based on an annual physical checkup or a school physical checkup within the previous 3 years. The present study included subjects $\geqslant 40$ years old with a normal ECG who were referred to our hospital because of chest pain or discomfort and were shown to be free from cardiovascular disease by multidetector computed tomography, myocardial scintigraphy or left heart catheterization. The present study also included subjects $<40$ years old with a normal ECG who were referred to our hospital because of chest pain or discomfort and were shown to be free from cardiovascular disease by exercise stress ECG. Subjects who were referred to our hospital because of bundle branch block or premature contractions and had normal echocardiographic findings, except for trivial valvular regurgitation, were also included. We prospectively enrolled eligible consecutive subjects who gave informed consent. These subjects also had normal findings based on an annual physical checkup. The present study was approved by the ethics committee of our institution (T10090), and all patients gave informed consent before their participation.

\section{Echocardiographic studies}

All echocardiographic studies were performed using an ACUSON Sequoia 512 ultrasound system (Siemens Medical Solutions, Mountain View, CA, USA) with a $4 \mathrm{~V} 1 \mathrm{c}$ transducer $(1.5-4.25 \mathrm{MHz})$. Measurements were made according to the criteria of the American Society of Echocardiography. ${ }^{4}$ The LV ejection fraction was obtained from the LV end-diastolic volumes and end-systolic volumes according to the biplane method using the modified version of Simpson's rule. LV mass was calculated at the end diastole. The volume and mass were indexed to the body surface area. Doppler measurements of mitral inflow E-wave and A-wave velocity were obtained, and tissue Doppler measurements of mitral e' wave velocity were made at the septal annulus.

After measurement of the standard echocardiographic parameters, a time-LAV curve was obtained by STE using offline software (Syngo Velocity Vector Imaging, Siemens Medical Solutions) to evaluate phasic LAV and LA function. As recommended by the American Society of Echocardiography, measurements of phasic LAV were made from the apical two- and fourchamber view. ${ }^{4}$ We also selected a measurement solely from the apical fourchamber view by STE because there were several poor recordings from the apical two-chamber view. Active LA EF measured by STE has been reported to be more accurate from the apical four-chamber view than the apical twochamber view compared with the current gold standard, three-dimensional CT $\left(r=0.73\right.$ for four-chamber view $v$ s. $r=0.45$ for two-chamber view). ${ }^{5}$ Total, passive and active LA EF were defined during a cardiac cycle as (maximum $\mathrm{LAV}$ - minimum $\mathrm{LAV}$ )/maximum $\mathrm{LAV} \times 100 \%$, (maximum LAV - pre-atrial contraction $\mathrm{LAV}$ )/maximum $\mathrm{LAV} \times 100 \%$ and (pre-atrial contraction $\mathrm{LAV}-$ minimum $\mathrm{LAV}) /$ pre-atrial contraction $\mathrm{LAV} \times 100 \%$, respectively. The LAV and function measurements were averaged from three consecutive beats. The reliability of the STE method for the quantification of phasic LAV and LA function has been well established in previous studies. ${ }^{6-8}$ The LA peak strain rate during systole, early diastole and atrial contraction were also obtained from the LA mid-septal and mid-lateral walls. The LA peak strain was obtained from the average of the LA mid-septal and mid-lateral wall strain. The E/e' was assessed as an index of diastolic function.

We recently reported that the logarithm of active LA EF/minimum LAV index was the best predictor of PCWP among all the ultrasound parameters based on multivariate regression analysis. The following regression equation was obtained from this analysis: PCWP $=10.8-12.4 \quad\left(\log _{10}\right.$ (active LA $\mathrm{EF} /$ minimum LAVI)) (measurements obtained from the apical four-chamber view). ${ }^{3}$ The ePCWP measured by this function was strongly correlated with the PCWP obtained by right-heart catheterization $(r=0.92)$ in patients in normal sinus rhythm (Figure 1). ${ }^{3}$ We named this novel index $\left(\log _{10}\right.$ (active LA $\mathrm{EF} /$ minimum LAVI)) the 'kinetics-tracking index: KT index'. The details of this index have been shown in a previous report. ${ }^{3}$ Briefly, we employed the LA volume as the denominator in the KT index to evaluate PCWP because as the LV diastolic function decreases, the LA volume increases. ${ }^{9,10}$ We determined the logarithmic correlation between the ePCWP and LA function using the LA function as the numerator in the KT index. Hsiao et al. ${ }^{10}$ previously reported a good logarithmic correlation between the LV filling pressure and the LA distensibility ((maximum LAVI - minimum LAVI)/minimum LAVI) that was similar to the total LA EF ((maximum LAVI-minimum LAVI)/maximum LAVI). Chamber stiffness is generally defined as a change in pressure relative to a change in volume of a hollow elastic structure. ${ }^{11}$ To estimate the stiffness in the LA, we used a change in the ePCWP as a substitute for a change in the LA pressure. We also used a change in the LA peak strain as a substitute for a change in the LA volume. Thus, we substituted ePCWP/LA peak strain for LA pressure and volume to determine LA stiffness.

\section{Reproducibility of PCWP estimated using the KT index}

We previously determined the interobserver variability of ePCWP in 30 patients with chest pain or discomfort. In that study, 30 randomly selected recordings were measured by two observers in a blinded manner. Similarly, we determined the intraobserver variability of the ePCWP in the same 30 recordings that were measured twice by one observer within a 7-day interval.

The intraobserver correlation coefficient and variability in the ePCWP were 0.99 and $0.9 \pm 1.5 \%$, respectively. The interobserver correlation coefficient and variability in the ePCWP were 0.99 and $2.5 \pm 1.8 \%$, respectively.

\section{Statistical analyses}

The data are expressed as the mean \pm s.d. The categorical data are summarized as percentages and compared using the $\chi^{2}$ test. Comparisons of parameters among eight groups were performed using two-way analysis of variance followed by Bonferroni's test for post hoc comparisons. Simple linear regression analysis was performed to determine the relationship among the echocardiographic parameters. Multivariate regression analysis was used to elucidate the relationships among the echocardiographic parameters and age. These statistical analyses were performed using Stat View version 5.0 (SAS Institution, Cary, NC, USA). Significant differences in the slopes of regression lines between males and females were tested using analysis of covariance for parallel slopes (Statflex version 6.0, Artech, Osaka, Japan). A $P$-value of $<0.05$ was considered significant.

\section{RESULTS}

\section{Characteristics of the study population and echocardiographic parameters}

We enrolled 198 healthy subjects. The characteristics of the study population and echocardiographic parameters are listed in Table 1 and Figure 1, respectively. The systolic blood pressure was within normal range $(126 \pm 8 \mathrm{~mm} \mathrm{Hg}$ in males and $125 \pm 9 \mathrm{~mm} \mathrm{Hg}$ in females) and increased with advancing age. The LV ejection fraction and the LV mass index were not significantly different among the eight age groups of males and females. The maximum, minimum and pre-atrial contraction LAVI significantly increased with advancing age, resulting in a deterioration of total and passive LA EF. E/A and E/e' (indicators of LV diastolic function) and LA stiffness significantly deteriorated with advancing age. However, the ePCWP was maintained because of compensation by an increase in active LA EF.

\section{Impact of healthy aging and gender on echocardiographic parameters}

Table 2 shows the correlation coefficients between the clinical and echocardiographic parameters and age. We selected body surface area and systolic blood pressure as clinical parameters to include in the multivariate regression analysis. We also selected E/e' and E/A as echocardiographic parameters to include in the multivariate regression analysis because these parameters have been demonstrated to be robust parameters that are associated with aging. We also selected active LA EF and minimum LAVI as echocardiographic parameters to include in the multivariate analysis because these parameters were the main focus of the present study. Multivariate regression analysis showed that systolic blood pressure, E/e', E/A, active LA EF and minimum LAVI were independently associated with age in males 

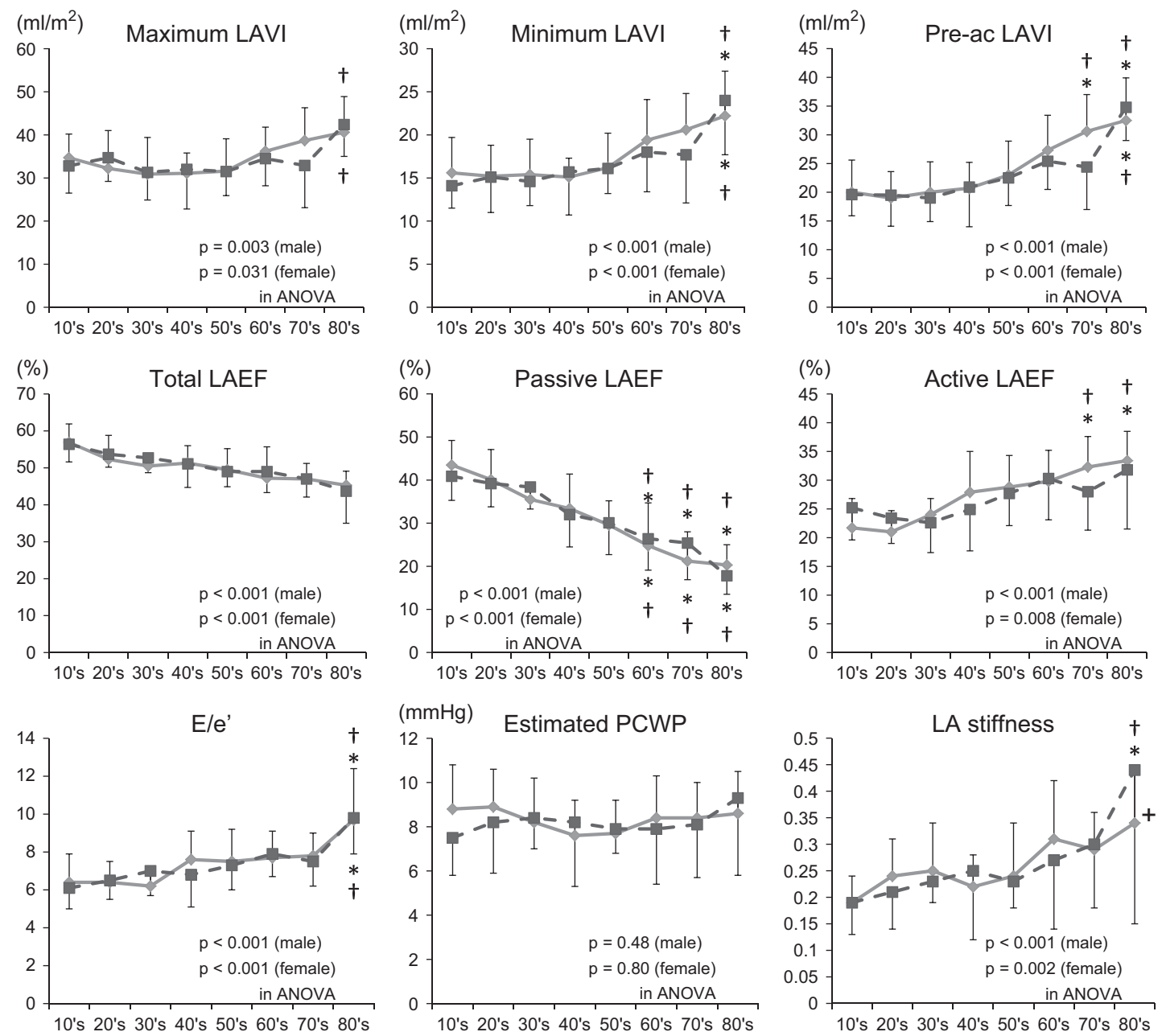

Figure 1 Left atrial volume and function for each decade. Solid line indicates male and broken line indicates female. ANOVA, analysis of variance; LA EF, left atrial emptying function; LAVI, left atrial volume index; PCWP, pulmonary capillary wedge pressure; Pre-ac, pre-atrial contraction. ${ }^{*} P<0.05$ vs. 20 ; ${ }^{\dagger} P<0.05$ vs. 30s, 40s and 50s; ${ }^{+} P<0.05$ vs. 40s. A full color version of this figure is available at the Hypertension Research journal online.

$\left(R^{2}=0.787\right.$; Table 3$)$. Similarly, multivariate regression analysis showed that E/e', E/A, active LA EF and minimum LAVI were independently associated with age in females $\left(R^{2}=0.809\right.$; Table 3$)$. The maximum and minimum LAVI was greater in male and female octogenarians than in males and females in their $30 \mathrm{~s}, 40 \mathrm{~s}$ and $50 \mathrm{~s}$ (Figure 1).

\section{Relationship between age and LV DD}

Figure 2 shows the relationships between age and echocardiographic parameters, indicating LV DD. With an increase in age, echocardiographic parameters, such as E/A and E/e' (indicators of LV diastolic function), deteriorated to the same extent in males and females (the slope of E/A was - 0.021 in males and -0.021 in females, and the slope of E/e' was 0.041 in males and 0.039 in females). However, there was no significant relationship between ePCWP and age. This finding suggested that ePCWP was maintained at $\sim 8 \mathrm{~mm} \mathrm{Hg}$ because of compensation by an increase in active LA EF in these healthy subjects. In contrast, the compensation for $\mathrm{LV} \mathrm{DD}$ by an increase in active LA EF tended to be more gradual in females (slope $=0.11$ ) than in males (slope $=0.18, P=0.060$ vs. females). Figure 3 shows the relationships between active LA EF and indicators of LV DD (that is, E/A and E/e') after adjusting for age. The correlation coefficients between active LA EF and E/A were stronger in males than in females $(-0.51 v s .-0.27, P=0.047)$. The slopes of these two relationships (regression lines) indicated that the strength of the compensation for LV dysfunction tended to be greater in males than in females, but this difference was not significant $(-5.74$ vs. -3.91 , $P=0.17$; Figure 3).

\section{DISCUSSION}

We determined the impact of gender and healthy aging on ePCWP using the KT index and other echocardiographic parameters that indicated LV DD in healthy subjects. The echocardiographic parameters that indicated LV DD deteriorated with advancing age in these healthy subjects. However, PCWP may be maintained because of compensation by an increase in active LA EF in both males and females. Furthermore, we demonstrated that the age-related compensation because of an increase in active LA EF was more gradual in females than in males, and this compensation in female septuagenarians and octogenarians was much weaker than in male septuagenarians and octogenarians.

The clinical value of estimated PCWP in healthy subjects

Recent advances in echocardiography have enabled the evaluation of LA function in patients with various diseases. ${ }^{8}$ We previously reported that hypertension is associated with an impairment of LA function and 
Table 1 Study population and echocardiographic parameters

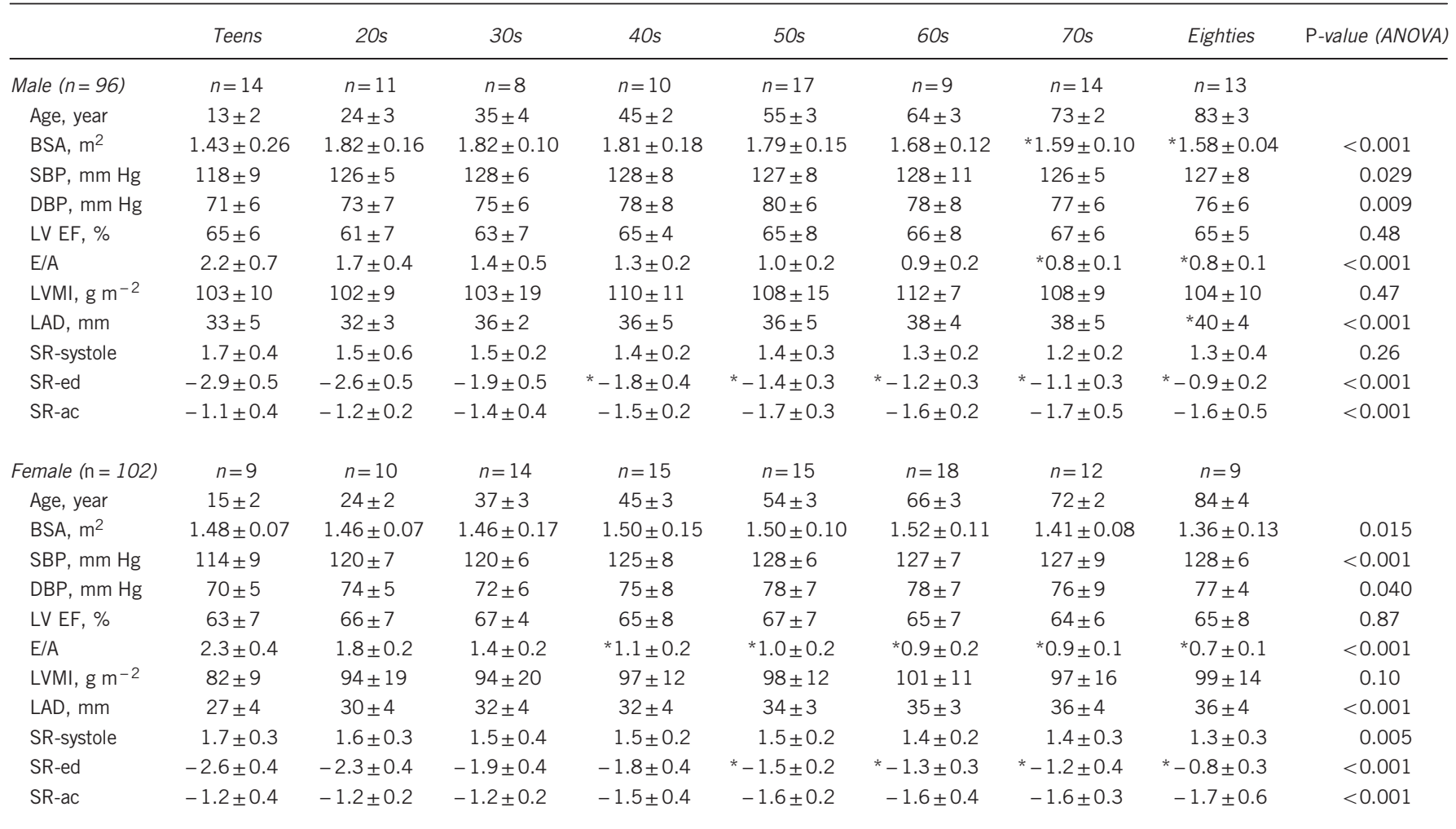

Abbreviations: ac, atrial contraction; ANOVA, analysis of variance; BSA, body mass index; DBP, diastolic blood pressure; E/A, ratio of the early to late diastolic filling velocity; ed, end-diastole; LAD, Abbreviations: ac, atrial contraction; ANOVA, analysis of variance; BSA, body mass index; DBP, diastolic blood pressure; E/A, ratio of the eaty
left atrial diameter; LV EF, left ventricular ejection fraction; LVMI, left ventricular mass index; SBP, systolic blood pressure; SR, strain rate. left atrial diameter
$* P<0.05$ vs. 20 s.

the risk of atrial fibrillation as assessed using STE. ${ }^{12}$ Hypertension affects many conditions of LV fluid volume and pressure status. For instance, anemia is associated with elevated LV filling pressure only in patients with hypertension but not in patients without hypertension. ${ }^{13}$ The noninvasive measurement of PCWP in healthy subjects and in patients with hypertension is valuable for the quantitative stratification of intravascular fluid volume and pressure status for the prediction of HF risk because of hypertension. However, there have been no previous studies evaluating PCWP in healthy subjects in a relatively large population because the measurement of PCWP requires an invasive method. The KT index we recently developed has a strong correlation with PCWP as measured by right-heart catheterization. ${ }^{3}$ In the present study, PCWP in healthy subjects was maintained regardless of deterioration of LV DD because of compensation by an increase in active LA EF. Daimon et al. ${ }^{14}$ reported that LV DD evaluated by E/A and E/e' deteriorated with advancing age in healthy Japanese subjects. However, that report did not evaluate PCWP. Fukuda et al. ${ }^{15}$ also reported echocardiographic parameters using three-dimensional echocardiography in healthy Japanese subjects. However, they focused only on volumetric parameters in the left atrium. In contrast, the present study focused on LA function, PCWP and LA volume, and demonstrated that the deterioration of LV DD because of aging was compensated by LA function; thus, PCWP was maintained.

\section{PCWP in healthy subjects}

There have been a few studies that have evaluated PCWP using healthy volunteers in small sample sizes. PCWP was measured by right-heart catheterization in 70 healthy volunteers. The PCWP in these subjects was $\sim 12 \mathrm{~mm} \mathrm{Hg}$ and did not differ among young ( $28 \pm 4$ years old, $57 \%$ men), early middle age ( $42 \pm 5$ years old, $32 \%$ men), late middle age $(57 \pm 4$ years old, $48 \%$ men) and seniors ( $70 \pm 3$ years old, $50 \%$ men), ${ }^{16}$ whereas the E/A ratio deteriorated from 1.9 to 0.8 . Although these findings were consistent with the present results, PCWP measured by right-heart catheterization $(12 \mathrm{~mm} \mathrm{Hg})$ was slightly higher than PCWP in the present study $(8 \mathrm{~mm} \mathrm{Hg})$. This discrepancy may be because measurement of PCWP by right-heart catheterization was performed using an invasive method and imposed a physical burden on the subjects, whereas measurement of ePCWP by STE in the present study did not impose a physical burden on the subjects. Another study reported PCWP measured by right-heart catheterization in 50 healthy, elderly, nonsedentary volunteers who were free of cardiovascular, pulmonary and systemic diseases, chest pain or shortness of breath and had normal electrocardiogram and echocardiographic findings. The PCWP in these subjects was $\sim 9 \mathrm{~mm} \mathrm{Hg}(65 \pm 10$ years old, $74 \% \mathrm{men}) .{ }^{17}$ This value was similar to the ePCWP obtained in the present study.

\section{Estimated PCWP compared with E/e'}

The E/e' has been proposed to estimate the LV filling pressure. ${ }^{18-20}$ In the present study, the E/A and E/e' ratios (indicators of LV diastolic function) significantly deteriorated with advancing age. However, the ePCWP was maintained at $\sim 8 \mathrm{~mm} \mathrm{Hg}$ because of compensation by an increase in active LA EF in these healthy subjects. These findings suggested that E/e' does not necessarily reflect PCWP. Estimation of PCWP or LV filling pressure using E/e' in healthy subjects or patients with mild LV diastolic function may not be valid because of the 
Table 2 Univariate regression analysis for the independent determinants of age

\begin{tabular}{|c|c|c|}
\hline & \multicolumn{2}{|c|}{ Univariate regression } \\
\hline & r & P-value \\
\hline \multicolumn{3}{|l|}{ Male } \\
\hline LV mass index & 0.10 & 0.32 \\
\hline LV ejection fraction & 0.16 & 0.11 \\
\hline$E / e^{\prime}$ & 0.51 & $<0.001$ \\
\hline$E / A$ & -0.80 & $<0.001$ \\
\hline Maximum LAVI & 0.29 & 0.005 \\
\hline Minimum LAVI & 0.47 & $<0.001$ \\
\hline Pre-ac LAVI & 0.60 & $<0.001$ \\
\hline Total LA EF & -0.56 & $<0.001$ \\
\hline Passive LA EF & -0.80 & $<0.001$ \\
\hline Active LA EF & 0.65 & $<0.001$ \\
\hline Estimated PCWP & -0.09 & 0.40 \\
\hline LA stiffness & 0.43 & $<0.001$ \\
\hline \multicolumn{3}{|l|}{ Female } \\
\hline LV mass index & 0.28 & 0.004 \\
\hline LV ejection fraction & 0.002 & 0.98 \\
\hline E/e' & 0.51 & $<0.001$ \\
\hline$E / A$ & -0.86 & $<0.001$ \\
\hline Maximum LAVI & 0.26 & 0.009 \\
\hline Minimum LAVI & 0.46 & $<0.001$ \\
\hline Pre-ac LAVI & 0.55 & $<0.001$ \\
\hline Total LA EF & 0.56 & $<0.001$ \\
\hline Passive LA EF & 0.71 & $<0.001$ \\
\hline Active LA EF & 0.32 & 0.001 \\
\hline Estimated PCWP & 0.13 & 0.21 \\
\hline LA stiffness & 0.43 & $<0.001$ \\
\hline
\end{tabular}

Abbreviations: $\mathrm{E} / \mathrm{A}$, ratio of the early to late diastolic filling velocity; E/e', ratio of early diastolic transmitral inflow velocity to annular tissue velocity; LA, left atrial; LA EF, left atrial emptying
function; LAVI, left atrial volume index; LV, left ventricular; PCWP, pulmonary capillary wedge pressure; Pre-ac, pre-atrial contraction.

compensation owing to an increase in active LA EF. The increase in active LA EF plays an important role in preventing an increase in PCWP with an increase in LV end-diastolic pressure.

Differences in atrial function between males and females

Several studies have demonstrated a difference in cardiac function between males and females. A recent study showed there was a significant relationship between cardiac and lung function in females after adjusting for confounding variables, whereas there was no such relationship in males. ${ }^{21}$ The maximum LAVI in octogenarians was greater than males and females in their 30s, 40s and 50s whereas deterioration of passive LA EF developed nearly 2 decades earlier in males and females. These results were consistent with the results obtained in a previous study. ${ }^{22}$ However, the present study showed compensation because of the increase in active LA EF only in male septuagenarians and octogenarians. There was also an intriguing finding in the present study. With an increase in age, echocardiographic parameters such as E/A and E/e', which reflect LV DD, deteriorated to the same extent in males and females. However, compensation for LV DD caused by an increase in active LA EF was less pronounced in females (slope $=0.11$ ) than in males (slope $=0.18$ ). In males, there was a strong correlation between active LA EF and E/A and between active LA EF and E/e' compared with females. The slopes of these two regression lines indicated that the strength of the
Table 3 Multivariate regression analysis for the independent determinants of age

\begin{tabular}{|c|c|c|c|}
\hline & Regression coefficient ( $\beta$ ) & P-value & Coefficient of determination $\left(\mathrm{R}^{2}\right)$ \\
\hline Male & & & 0.787 \\
\hline $\begin{array}{l}\text { Body surface } \\
\text { area }\end{array}$ & - & 0.93 & \\
\hline $\begin{array}{l}\text { Systolic blood } \\
\text { pressure }\end{array}$ & 0.118 & 0.021 & \\
\hline E/e' & 0.117 & 0.046 & \\
\hline $\mathrm{E} / \mathrm{A}$ & -0.526 & $<0.001$ & \\
\hline Active LA EF & 0.290 & $<0.001$ & \\
\hline Minimum LAVI & 0.197 & $<0.001$ & \\
\hline Female & & & 0.809 \\
\hline $\begin{array}{l}\text { Body surface } \\
\text { area }\end{array}$ & - & 0.86 & \\
\hline $\begin{array}{l}\text { Systolic blood } \\
\text { pressure }\end{array}$ & - & 0.20 & \\
\hline$E / e^{\prime}$ & 0.065 & 0.013 & \\
\hline$E / A$ & -0.691 & $<0.001$ & \\
\hline Active LA EF & 0.107 & 0.042 & \\
\hline Minimum LAVI & 0.168 & 0.004 & \\
\hline
\end{tabular}

compensation for LV DD was also greater in males than in females. As shown in Figure 3, compensation because of an increase in active LA EF was more prominent in males than in females. The Framingham Heart Study showed that the prevalence of congestive HF in septuagenarians was higher in males than in females. ${ }^{23}$ However, the prevalence of congestive HF in octogenarians was higher in females than in males. The 11-year follow-up of the PREVEND study showed that female gender was associated with new-onset HF with preserved LV ejection fraction, whereas male gender was associated with newonset HF with reduced LV ejection fraction after adjusting for age. ${ }^{24}$ In the present study, compensation for LV DD caused by an increase in active LA EF was significantly increased only in male octogenarians compared with female octogenarians. This finding may explain why HF with preserved LV ejection fraction occurs more frequently in females than in males.

It has recently been reported that hormone replacement therapy by estrogen and/or progestogen reduced new-onset atrial fibrillation in the first year after myocardial infarction. ${ }^{25}$ The underlying mechanisms remain unknown. Together with the results of the present study, it is possible that a deficiency in female hormones may impair diastolic function.

\section{Clinical implications}

The potential clinical application of the present methods and findings is broad. Noninvasive measurement of ePCWP is useful for the evaluation of intravascular fluid volume and pressure status for the onset of HF. Risk stratification of HF using PCWP is important for understanding when and why HF develops in patients and healthy subjects. This method may also be applicable for the determination of appropriate dry weight during dialysis and prediction of the onset of atrial fibrillation. A recent study demonstrated that increases in active LA EF during leg lifts were blunted in HF patients with preserved LV ejection fraction, and this resulted in a reduction in LA stroke volume. ${ }^{26}$ Regarding ePCWP, further investigations are necessary to 


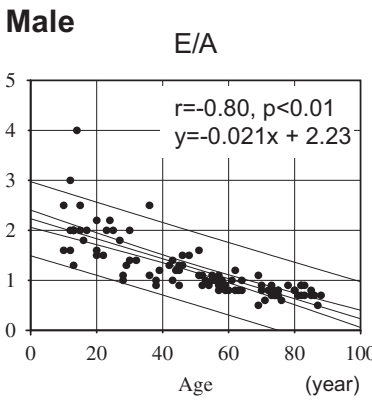

Female

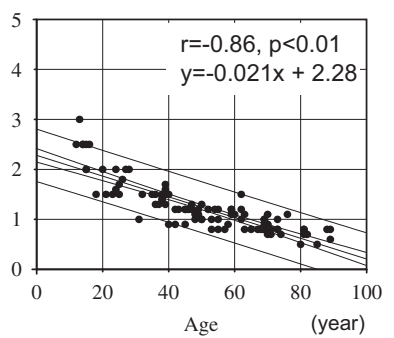

$E / e^{\prime}$

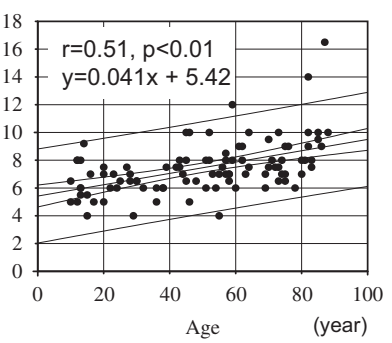

$E / e^{\prime}$

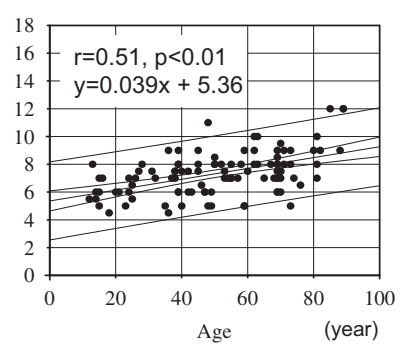

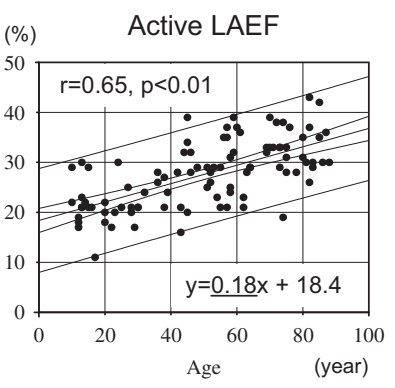
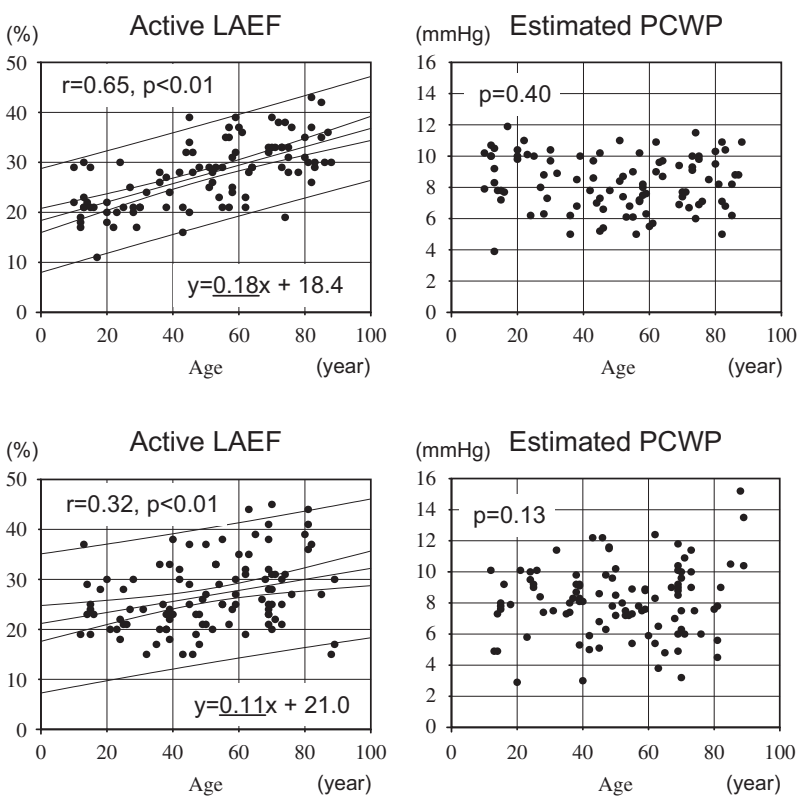

Figure 2 Relationships between age and echocardiographic parameters. LA EF, left atrial emptying function; PCWP, pulmonary capillary wedge pressure. Center line indicates the regression line. Inner lines indicate the $95 \%$ confidential intervals of the regression line. Outer lines indicate the $95 \%$ confidential interval of the raw data.
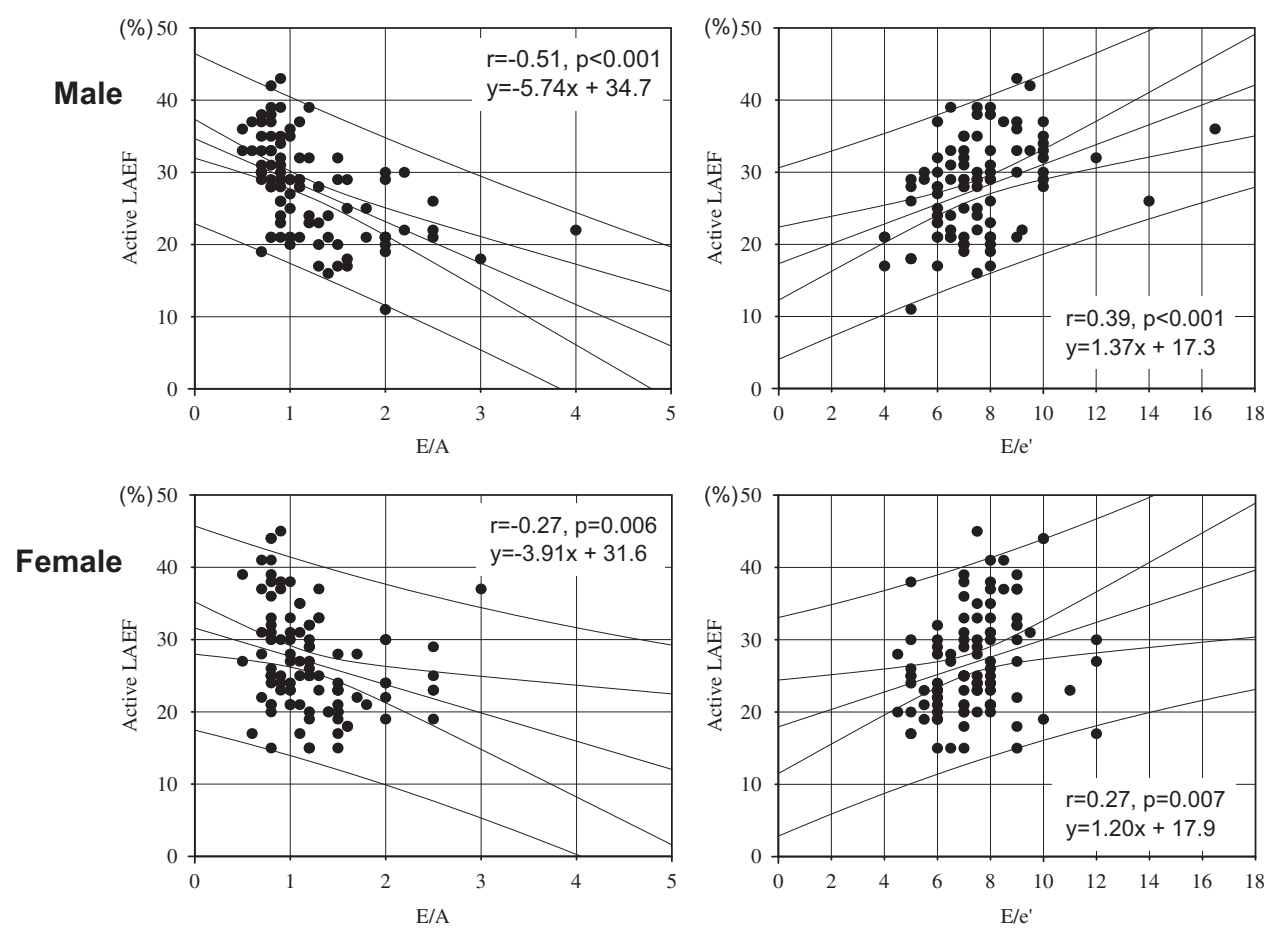

Figure 3 Relationships between active left atrial emptying function (LA EF) and echocardiographic parameters. Center line indicates the regression line. Inner lines indicate the $95 \%$ confidential intervals of the regression line. Outer lines indicate the $95 \%$ confidential interval of the raw data.

evaluate the leg lifts on ePCWP to determine whether these changes may have value in HF risk stratification.

\section{Study limitations}

There are several limitations of the present study. First, we evaluated only a small number of subjects in each age group, whereas the number of total healthy subjects was large. In addition, the present study was based on a two-dimensional measurement. Threedimensional STE is the most recent technique and is now available to assess LA structure and function, including LA volume and strain rate, without any assumptions of LA geometry. ${ }^{2}$ Reassessment of the values in the present study using three-dimensional STE will be required in the future. ${ }^{27,28}$ Second, variation of intravascular fluid volume and pressure status due to dehydration may be inevitable even 
in healthy subjects. In addition, there was a possibility that we overlooked subjects $<40$ years old with cardiovascular disease because we performed only an exercise stress ECG in our subjects. Third, the 95\% limits of agreement in the Bland-Altman plot between PCWP that was measured by right-heart catheterization and KT index was $\pm 4.05 \mathrm{~mm} \mathrm{Hg} \quad( \pm 1.96$ s.d. $)$ in patients with normal PCWP $(\leqslant 12 \mathrm{~mm} \mathrm{Hg})$ in our previous study. ${ }^{3}$ These limits of agreement were relatively wide. However, when the variation of the values above the regression line is the same as that below the regression line, the average of the values becomes closer to the true value because of 'regression toward the mean.' In addition, comparison of the PCWP measured by right-heart catheterization and the KT index was not performed in healthy subjects because right-heart catheterization could not be performed owing to ethical reasons. This limitation hindered the rigorous validation of the impact of gender and healthy aging on PCWP.

\section{CONCLUSIONS}

The echocardiographic parameters that indicated LV DD deteriorated with advancing age in healthy subjects. PCWP may be maintained because of compensation by an increase in active LA EF in both males and females. This compensation tended to be more gradual in females than males, and the compensation in female septuagenarians and octogenarians was weaker than in male septuagenarians and octogenarians.

\section{CONFLICT OF INTEREST}

The authors declare no conflict of interest.

\section{ACKNOWLEDGEMENTS}

This research did not receive a grant from any funding agency in the public, commercial or not-for-profit sectors.

1 Baicu CF, Zile MR, Aurigemma GP, Gaasch WH. Left ventricular systolic performance, function, and contractility in patients with diastolic heart failure. Circulation 2005; 111: 2306-2312.

2 Saeki M, Sato N, Kawasaki M, Tanaka R, Nagaya M, Watanabe T, Ono K, Noda T, Zile MR, Minatoguchi S. Left ventricular layer function in hypertension assessed by myocardial strain rate using novel one-beat real-time three-dimensional speckle tracking echocardiography with high volume rates. Hypertens Res 2015; 38: 551-559.

3 Kawasaki M, Tanaka R, Ono K, Minatoguchi S, Watanabe T, Iwama M, Hirose T, Arai M, Noda T, Watanabe S, Zile MR, Minatoguchi S. A novel ultrasound predictor of pulmonary capillary wedge pressure assessed by the combination of left atrial volume and function: a speckle tracking echocardiography study. J Cardiol 2015; 66 : 253-262.

4 Lang RM, Bierig M, Devereux RB, Flachskampf FA, Foster E, Pellikka PA, Picard MH Roman MJ, Seward J, Shanewise JS, Solomon SD, Spencer KT, Sutton MS, Stewart WJChamber Quantification Writing GroupAmerican Society of Echocardiography's Guidelines and Standards CommitteeEuropean Association of Echocardiography. Recommendations for chamber quantification: a report from the American Society of Echocardiography's Guidelines and Standards Committee and the Chamber Quantification Writing Group, developed in conjunction with the European Association of Echocardiography, a branch of the European Society of Cardiology. J Am Soc Echocardiogr 2005; 18: 1440-1463.

5 Nagaya M, Kawasaki M, Tanaka R, Onishi N, Sato N, Ono K, Watanabe T, Minatoguchi S, Miwa H, Goto Y, Hirose T, Arai M, Noda T, Watanabe S, Minatoguchi S. Quantitative validation of left atrial structure and function by two-dimensional and three-dimensional speckle tracking echocardiography: a comparative study with three-dimensional computed tomography. J Cardiol 2013; 62: 188-194.

6 Ogawa K, Hozumi T, Sugioka K, Iwata S, Otsuka R, Takagi Y, Yoshitani H, Yoshiyama M, Yoshikawa J. Automated assessment of left atrial function from time-left atrial volume curves using a novel speckle tracking imaging method. J Am Soc Echocardiogr 2009; 22: 63-69.

7 Hirose T, Kawasaki M, Tanaka R, Ono K, Watanabe T, Iwama M, Noda T, Watanabe S, Takemura G. Minatoguchi S. Left atrial function assessed by speckle tracking echocardiography as a predictor of new-onset non-valvular atrial fibrillation: results from a prospective study in 580 adults. Eur Heart J Cardiovasc Imaging 2012; 13: 243-250.

8 Fang NN, Sui DX, Yu JG, Gong HP, Zhong M, Zhang Y, Zhang W. Strain/strain rate imaging of impaired left atrial function in patients with metabolic syndrome. Hypertens Res 2015; 38: 758-764.

9 Pritchett AM, Mahoney DW, Jacobsen SJ, Rodeheffer RJ, Karon BL, Redfield MM. Diastolic dysfunction and left atrial volume: a population-based study. J Am Coll Cardiol 2005; 45: 87-92.

10 Hsiao SH, Huang WC, Lin KL, Chiou KR, Kuo FY, Lin SK, Cheng CC. Left atria distensibility and left ventricular filling pressure in acute versus chronic severe mitral regurgitation. Am J Cardiol 2010; 105: 709-715.

11 Mirsky I. Assessment of diastolic function: suggested methods and future considerations. Circulation 1984; 69: 836-841.

12 Watanabe T, Kawasaki M, Tanaka R, Ono K, Nishigaki K, Takemura G, Arai M, Noda T, Watanabe S, Minatoguchi S. Association among blood pressure control in elderly patients with hypertension, left atrial structure and function and new-onset atrial fibrillation: a prospective 2-year study in 234 patients. Hypertens Res 2013; 36: 799-806.

13 Cho I, Kim MA, Shim WJ, Park SM, Kim YH, Na JO, Shin MS, Kim YJ, Yoon HJ, Cho YK, Kim SE, Hong KS, Kim HS, Cho KI, Shin GJ. Differential impact of anemia on left ventricular filling pressure between subjects with and without hypertension. Results from a multicenter, cohort study. J Hypertens 2015; 33 (Suppl 1): e105-e106.

14 Daimon M, Watanabe H, Abe Y, Hirata K, Hozumi T, Ishii K, Ito H, Iwakura K, Izumi C, Matsuzaki M, Minagoe S, Abe H, Murata K, Nakatani S, Negishi K, Yoshida K, Tanabe K, Tanaka N, Tokai K, Yoshikawa JJAMP Study Investigators. Normal values of echocardiographic parameters in relation to age in a healthy Japanese population: the JAMP study. Circ J 2008; 72: 1859-1866.

15 Fukuda S, Watanabe H, Daimon M, Abe Y, Hirashiki A, Hirata K, Ito H, Iwai-Takano M, Iwakura K, Izumi C, Hidaka T, Yuasa T, Murata K, Nakatani S, Negishi K, Nishigami K, Nishikage T, Ota T, Hayashida A, Sakata K, Tanaka N, Yamada S, Yamamoto K, Yoshikawa J. Normal values of real-time 3-dimensional echocardiographic parameters in a healthy Japanese population: the JAMP-3D Study. Circ J 2012; 76: 1177-1181.

16 Carrick-Ranson G, Hastings JL, Bhella PS, Shibata S, Fujimoto N, Palmer MD, Boyd K, Levine BD. Effect of healthy aging on left ventricular relaxation and diastolic suction. Am J Physiol Heart Circ Physiol 2012; 303: H315-H322.

17 Guron CW, Persson A, Wikh R, Caidahl K. Can the left ventricular early diastolic tissueto-blood time interval be used to identify a normal pulmonary capillary wedge pressure? Eur J Echocardiogr 2007; 8: 94-101.

18 Nagueh SF, Middleton KJ, Kopelen HA, Zoghbi WA, Quinones MA. Doppler tissue imaging: a noninvasive technique for evaluation of left ventricular relaxation and estimation of filling pressures. J Am Coll Cardiol 1997; 30: 1527-1533.

19 Nagueh SF, Mikati I, Kopelen HA, Middleton KJ, Quinones MA, Zoghbi WA. Doppler estimation of left ventricular filling pressure in sinus tachycardia: a new application of tissue Doppler imaging. Circulation 1998; 98: 1644-1650.

20 Nagueh SF, Bhatt R, Vivo RP, Krim SR, Sarvari SI, Russell K, Edvardsen T, Smiseth OA, Estep JD. Echocardiographic evaluation of hemodynamics in patients with decompensated systolic heart failure. Circ Cardiovasc Imaging 2011; 4: 220-227.

21 Mendes PR, Kiyota TA, Cipolli JA, Schreiber R, Paim LR, Bellinazzi VR, Matos-Souza JR, Sposito AC, Nadruz W Jr. Gender influences the relationship between lung function and cardiac remodeling in hypertensive subjects. Hypertens Res 2015; 38: 264-268.

22 Boyd AC, Schiller NB, Leung D, Ross DL, Thomas L. Atrial dilation and altered function are mediated by age and diastolic function but not before the eighth decade. JACC Cardiovasc Imaging 2011; 4: 234-242.

23 Pitt B. Part II: new insights into the epidemiology and pathophysiology of heart failure. $J$ Am Coll Cardiol 1993; 22: 6A-13A.

24 Brouwers FP, de Boer RA, van der Harst P, Voors AA, Gansevoort RT, Bakker SJ, Hillege HL, van Veldhuisen DJ, van Gilst WH. Incidence and epidemiology of new onset heart failure with preserved vs. reduced ejection fraction in a community-based cohort: 11-year follow-up of PREVEND. Eur Heart J 2013; 34: 1424-1431.

25 Bretler DM, Hansen PR, Lindhardsen J, Ahlehoff O, Andersson C, Jensen TB, Rauns $\varnothing$ J Torp-Pedersen C, Gislason GH. Hormone replacement therapy and risk of new-onset atrial fibrillation after myocardial infarction-a nationwide cohort study. PLOS ONE 2012; 7 (12): e51580.

26 Obokata M, Negishi K, Kurosawa K, Arima H, Tateno R, Ui G, Tange S, Arai M, Kurabayashi M. Incremental diagnostic value of la strain with leg lifts in heart failure with preserved ejection fraction. JACC Cardiovasc Imaging 2013; 6: 749-758.

27 Onishi N, Kawasaki M, Tanaka R, Sato H, Saeki M, Nagaya M, Sato N, Minatoguchi S, Watanabe T, Ono K, Arai M, Noda T, Amano K, Goto K, Watanabe S, Minatoguchi S. Comparison between left atrial features in well-controlled hypertensive patients and normal subjects assessed by three-dimensional speckle tracking echocardiography. $J$ Cardiol 2014; 63: 291-295.

28 Kawamura R, Seo Y, Ishizu T, Atsumi A, Yamamoto M, Machino-Ohtsuka T, Nakajima H, Sakai S, Tanaka YO, Minami M, Aonuma K. Feasibility of left ventricular volume measurements by three-dimensional speckle tracking echocardiography depends on image quality and degree of left ventricular enlargement: validation study with cardiac magnetic resonance imaging. J Cardiol 2014; 63: 230-238. 